\title{
Operation Gritrock: first UK army medics fly to Sierra Leone
}

After comprehensive training, 150 army medics are ready to help fight the Ebola outbreak in Africa. Andy Johnston and Mark Bailey describe the deployment

\author{
Andy Johnston consultant in respiratory and intensive care medicine, Royal Centre for Defence \\ Medicine, Queen Elizabeth Hospital, Birmingham, Mark Bailey consultant in infectious diseases, \\ Heartlands Hospital, Birmingham
}

This week the first group of 150 British army medical staff fly to Sierra Leone to set up a treatment centre for health workers infected with the Ebola virus. We hope that the provision of high quality medical care will reduce mortality among affected patients. Our facility will be sited next to a larger Ebola treatment centre to be run by the charity Save the Children.

We have spent the past two weeks in comprehensive training, including lectures from experts in infectious disease, narrative accounts from health workers who have been to Sierra Leone, cultural awareness and language training, and hands-on "whole hospital" simulation. The Army Medical Services Training Centre, near York, has been used for the past decade to train field hospitals deploying to Afghanistan, using high fidelity simulation with electronic manikins, actors, and a hospital laid out identically to the one at Camp Bastion, the main British military base in Afghanistan. This has now been rebuilt as a tented facility, using the layout of a Médecins Sans Frontières Ebola treatment centre.

Experts in Ebola from Public Health England and specialists in chemical, biological, radiological, and nuclear (CBRN) medicine from the National Ambulance Resilience Unit and the Defence CBRN Centre have trained the 150 medical staff deploying to Sierra Leone in how to use the personal protective equipment needed to keep our staff safe and to prevent cross infection. Military engineers are already in Sierra Leone building the Ebola treatment centre in Kerry Town, near the southern end of the Freetown peninsula. The UK Department for International Development is providing the resources, including infusion pumps, fluids, drugs, and the vast quantities of personal protective equipment needed. The initial mission to build a single treatment centre has now expanded to one of providing further engineering, logistical, and medical input to create more Ebola treatment centres around the country.

Besides one of the authors (MB), the team includes Major Tom Fletcher, an infectious disease physician who has recently returned from treating patients infected with Ebola in Africa. Military intensive care specialists, internal medicine specialists, nurses, and administrators will be key members of the team. All the doctors and many of the nurses have worked overseas on military operations in Iraq or Afghanistan, and several of the infectious disease physicians have worked in tropical medicine centres in Asia and Africa. Our aspiration is to provide a high standard of healthcare, including investigations in an on-site laboratory, treatment of coinfection with malaria and other diseases, and correction of electrolyte and fluid balance disturbances, by using central venous access where necessary. Electronic scanning of the patients' records and collection of a standard dataset used in viral haemorrhagic fevers will allow rapid analysis and implementation of clinical lessons.

The treatment centre will run in the manner it would in a more conventional deployment, commanded by nursing officer Lieutenant Colonel Alison McCourt and with Lieutenant Colonel Dave Hinsley, an orthopaedic surgeon, as the medical director. Operations in Afghanistan have taught us that the hospital works well with an experienced commander and a medical director who provides clinical oversight of the hospital. Expert external support from Public Health England, the National Ambulance Resilience Unit, the World Health Organization, and other agencies assisted in the training and validation.

The epidemic seems likely to continue for many months, and as the first team of army medics deploy we are planning the training package for their replacements.

Competed interests: None declared.

Provenance and peer review: Commissioned; not externally peer reviewed.

Accepted: 13 Oct 2014

Cite this as: BMJ 2014;349:g6237

(c) BMJ Publishing Group Ltd 2014 